\title{
Necrotizing fasciitis arisen from nose
}

\author{
Inhoe Ku, Ji-Ung Park \\ Department of Plastic and Reconstructive Surgery, Seoul National University Boramae Hospital, Seoul National University College of Medicine, \\ Seoul, Korea
}

A 48-year-old man presented to the emergency room with a 6-day history of exacerbating erythema and swelling on the nose, upper lip, bilateral cheek, and periorbital area. He denied any facial trauma history but reported recent nasal hair plucking. There were necrotic gangrene and crepitus on the nose, upper lip and bilateral cheek with odorous purulent discharge (Fig. 1A). A contrast-enhanced computed tomography confirmed air opacities in soft tissues of the midface (Fig. 2). Overall vital status was unstable with body temperature reaching $39.7^{\circ} \mathrm{C}$. The lab results of the patient strongly suggested high risk of necrotizing fasciitis with the Laboratory Risk Indicator for Necrotizing Fasciitis (LRINEC) score 12. The patient was under poor hygiene and had history of alcohol abuse and uncontrolled diabetic mellitus with unknown onset.

Promptly broad-spectrum antibiotics combination of ampicillin/sulbactam and clindamycin were intravenously (IV) administrated, and initial debridement was performed under general anesthesia. Five days later tissue cultures grew Klebsiella pneumoniae and clindamycin-resistant group B Streptococcus. The antibiotics regimen was changed to ceftriaxone and metronidazole. The patient underwent serial debridement (Fig. 1B) until there was no pus-like discharge from the wound (Fig. 1C). After inflammatory signs and blood test normalized, the facial defect was successfully reconstructed with forehead flap, abbe flap, and bilateral cheek flaps combined (Fig. 1D).

Facial necrotizing fasciitis (NF) spreads rapidly along fascial planes causing extensive necrosis. Immediate surgical debride-

\section{Correspondence: Ji-Ung Park}

Department of Plastic and Reconstructive Surgery, Seoul National University Boramae Hospital, Seoul National University College of Medicine, 20 Boramae-ro 5-gil, Dongjak-gu, Seoul 07061, Korea

E-mail: alfbskan@gmail.com

Received May 23, 2019 / Revised June 25, 2019 / Accepted July 1, 2019 ment of the necrotic tissue and administration of broad-spectrum IV antibiotics are crucial to prevent associated mortality. Facial NF mostly originates from dental or pharyngeal abscess and radiotherapy $[1,2]$. However, unlike other anatomic sites
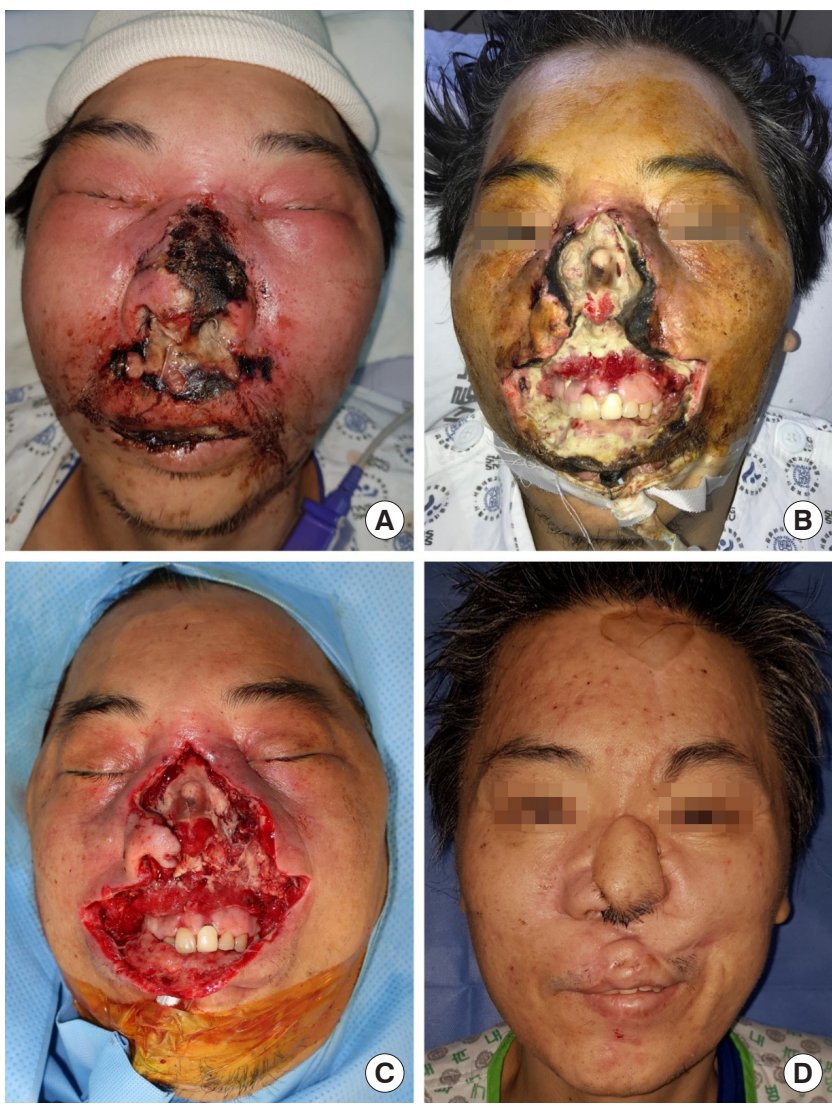

Fig. 1. Patient photos at (A) emergency room admission, (B) before 2nd serial debridement, $(\mathrm{C})$ after 3rd debridement confirming no purulent discharge and fresh soft tissue, (D) 3 months after reconstruction with forehead flap, abbe flap, and bilateral cheek flaps combined. 

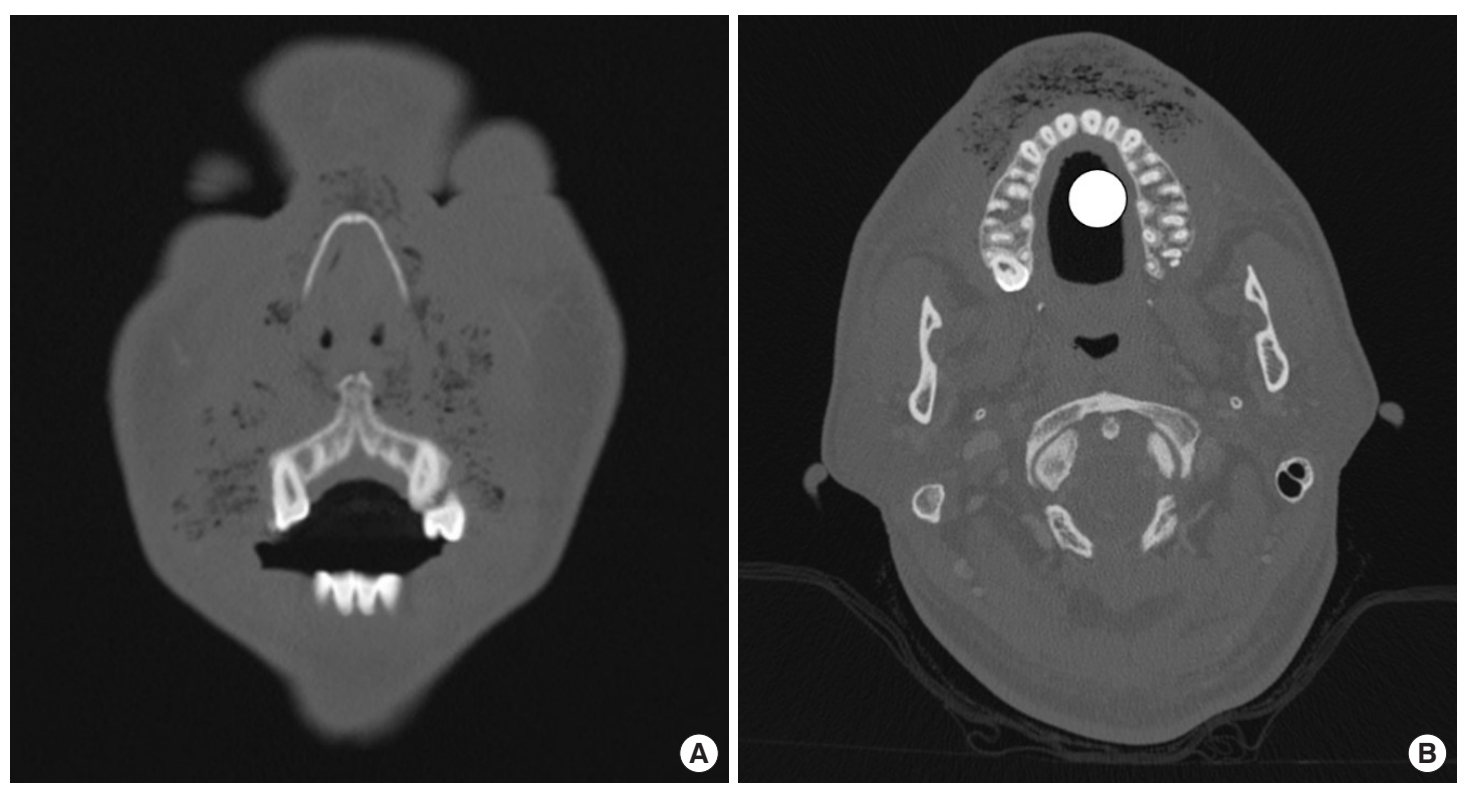

Fig. 2. (A, B) Craniofacial contrast-enhanced computed tomography at the patient's admission showing air opacities among bilateral cheek, upper lip, and nasal soft tissue.

facial region is rarely affected to NF with the aid of their rich vascular supply [3].

Herein, we report a rare case of a patient who was diagnosed with facial NF without recent history of dental abscess nor radiotherapy. Both nasal plucking and immunocompromised state with uncontrolled diabetic mellitus are predisposing factors for nasal vestibulitis, which may have affected the patient to be prone to the facial NF arisen from nose [4].

\section{NOTES}

\section{Conflict of interest}

No potential conflict of interest relevant to this article was reported.

\section{Ethical approval}

The study was approved by the Institutional Review Board of Seoul National University Boramae Hospital (IRB No. 30-20199) and performed in accordance with the principles of the Declaration of Helsinki. Written informed consent was obtained.

\section{Patient consent}

The patient provided written informed consent for the publication and the use of his images.

\section{ORCID}

Inhoe Ku https://orcid.org/0000-0002-3046-6800

Ji-Ung Park https://orcid.org/0000-0002-6403-4918

\section{REFERENCES}

1. Hernandez DA, Manuel A, Chavez G, Rivera AS. Facial necrotizing fasciitis in adults: a systematic review. Heighpubs Otolaryngol and Rhinol 2017;1:20-31.

2. Morgan MS. Recent advances in the treatment of necrotizing fasciitis. Curr Infect Dis Rep 2011;13:461-9.

3. Shindo ML, Nalbone VP, Dougherty WR. Necrotizing fasciitis of the face. Laryngoscope 1997;107:1071-9.

4. Lipschitz N, Yakirevitch A, Sagiv D, Migirov L, Talmi YP, Wolf $\mathrm{M}$, et al. Nasal vestibulitis: etiology, risk factors, and clinical characteristics: a retrospective study of 118 cases. Diagn Microbiol Infect Dis 2017;89:131-4. 